\title{
ON A GENERAL TAUBERIAN THEOREM
}

\author{
T. T. $\mathrm{MOH}$
}

ABSTRACT. In this article the celebrated Tauberian theorem by Norbert Wiener ([1], [2]) is considerably extended by the introduction of a wider class of translation kernels. A number of applications are given to show the fruitfulness of this new generalization.

Introduction. To begin with we quote the celebrated Tauberian theorem by Norbert Weiner ([1], [2]).

Assume that $K(x) \in L^{1}(-\infty, \infty), \varphi(x) \in L^{\infty}(-\infty, \infty)$ and let the Fourier transform of $K$ be $\neq 0$ for any real argument. The relation

$$
\lim _{x=\infty} \int_{-\infty}^{\infty} \varphi(\xi) K(x-\xi) d \xi=A \int_{-\infty}^{\infty} K(\xi) d \xi
$$

where $A$ is a number, implies for any $H(x) \in L^{1}(-\infty, \infty)$

$$
\lim _{x=\infty} \int_{-\infty}^{\infty} \varphi(\xi) H(x-\xi) d \xi=A \int_{-\infty}^{\infty} H(\xi) d \xi .
$$

It was also shown in the quoted paper that a large number of classical Tauberian theorems can be reduced to the result stated above concerning the two integrals. The scope of Wiener's theorem can, however, be considerably extended by the introduction of a wider class of translation kernels.

Let $\sigma(x)$ be a positive measurable function on the real axis satisfying the conditions:

$$
\begin{gathered}
\lim _{x=\infty} \frac{\sigma(x)}{x}=0 \\
\lim _{x=\infty} \frac{\sigma(x+t \sigma(x))}{\sigma(x)}=1
\end{gathered}
$$

valid for a.a. real $t$. Let $E$ be the set of $t$ such that (4) is valid. We shall prove this.

Received by the editors January 27, 1972 and, in revised form, February 18, 1972. AMS 1970 subject classifications. Primary 40E05.

Key words and phrases. Translation kernel, spectrum of $\psi$.

(c) American Mathematical Society 1972 
Theorem I. Let $K, H, \varphi$ and $A$ be as in Wiener's theorem and let $\sigma(x)$ satisfy the conditions stated above. Then

$$
\lim _{x=\infty} \int_{-\infty}^{\infty} \varphi(\xi) K\left(\frac{x-\xi}{\sigma(x)}\right) \frac{d \xi}{\sigma(x)}=A \int_{-\infty}^{\infty} K(\xi) d \xi
$$

implies

$$
\lim _{x=\infty} \int_{-\infty}^{\infty} \varphi(\xi) H\left(\frac{x-\xi}{\sigma(x)}\right) \frac{d \xi}{\sigma(x)}=A \int_{-\infty}^{\infty} H(\xi) d \xi
$$

This extension of Wiener's theorem was suggested to me by Professor Beurling and will be proved by a method used by him in proving Wiener's original result [1].

1. A proof of Theorem I. Replacing $\varphi(x)$ by $\varphi(x)-A_{2}$ we may assume $A=0$. Let

$$
f(x)=\int_{-\infty}^{\infty} K\left(\frac{x-\xi}{\sigma(x)}\right) \frac{1}{\sigma(x)} \varphi(\xi) d \xi, \quad g(x)=\int_{-\infty}^{\infty} H\left(\frac{x-\xi}{\sigma(x)}\right) \frac{1}{\sigma(x)} \varphi(\xi) d \xi .
$$

There exists a bounded continuous function $\alpha(h)$, with $\alpha(0)=0$, such that $K($ and $H$ ) satisfy

$$
\int_{-\infty}^{\infty}|K(\xi+h)-K(\xi)| d \xi \leqq \alpha(h) .
$$

Similarly $\omega(x)$ exists continuous and bounded with $\omega(1)=0, \omega(\lambda)=\omega(1 / \lambda)$, such that

$$
\int_{-\infty}^{\infty}\left|\frac{1}{\lambda} K\left(\frac{\xi}{\lambda}\right)-K(\xi)\right| d \xi \leqq \omega(\lambda) .
$$

It follows from $\left(1^{\prime}\right)$ that $\lim _{x=\infty} f(x)=0$. Suppose the theorem is false; then there exists a sequence $\left\{x_{n}\right\} \nearrow \infty$ such that $\left|g\left(x_{n}\right)\right| \geqq d>0$. Let

$$
f_{n}(x)=f\left(x_{n}+\sigma\left(x_{n}\right) x\right), \quad g_{n}(x)=g\left(x_{n}+\sigma\left(x_{n}\right) x\right) .
$$

As a first step we shall prove that

$$
\lim _{n=\infty}\left\{K * g_{n}(x)-H * f_{n}(x)\right\}=0,
$$

where $*$ denotes convolution. To prove (7) it is enough to prove

$$
\begin{aligned}
\lim _{n=\infty} \mid K * g_{n}(x)-\int_{-\infty}^{\infty} & \int_{-\infty}^{\infty} K(x-\xi) H(x-\eta) \\
& \cdot \varphi\left(x_{n}+\xi \sigma\left(x_{n}\right)+\eta \sigma\left(x_{n}\right)-x \sigma\left(x_{n}\right)\right) d \xi d \eta \mid=0
\end{aligned}
$$


since the second term is symmetric in $\xi$ and $\eta$. Let $\xi^{\prime}=x_{n}+\sigma\left(x_{n}\right) \xi$. Then

$$
\begin{aligned}
& \lim _{n=\infty} \mid \int_{-\infty}^{\infty} \int_{-\infty}^{\infty} K(x-\xi) H\left(\frac{\xi^{\prime}-\eta}{\sigma\left(\xi^{\prime}\right)}\right) \frac{1}{\sigma\left(\xi^{\prime}\right)} \varphi(\eta) d \xi d \eta \\
& \quad-\int_{-\infty}^{\infty} \int_{-\infty}^{\infty} K(x-\xi) H(x-\eta) \varphi\left(\xi^{\prime}-(x-\eta) \sigma\left(x_{n}\right)\right) d \xi d \eta \mid \\
& =\lim _{n=\infty} \mid \int_{-\infty}^{\infty} \int_{-\infty}^{\infty} K(x-\xi) \varphi(\eta) H\left(\frac{\xi^{\prime}-\eta}{\sigma\left(\xi^{\prime}\right)}\right) \frac{1}{\sigma\left(\xi^{\prime}\right)} d \xi d \eta \\
& \leqq\|\varphi(\eta)\|_{L^{\infty}} \lim _{n=\infty} \int_{-\infty}^{\infty} \int_{-\infty}^{\infty}|K(x-\xi)| \\
& \quad \times\left|H\left(\frac{\xi^{\prime}-\eta}{\sigma\left(\xi^{\prime}\right)}\right) \frac{1}{\sigma\left(\xi^{\prime}\right)}-H\left(\frac{\xi^{\prime}-\eta}{\sigma\left(x_{n}\right)}\right) \frac{1}{\sigma\left(x_{n}\right)}\right| d \eta d \xi \\
& \leqq\|\varphi(\eta)\|_{L^{\infty}} \lim _{n=\infty} \int_{-\infty}^{\infty}|K(x-\xi)| \omega\left(\frac{\sigma\left(x_{n}\right)}{\sigma\left(\xi^{\prime}\right)}\right) d \xi .
\end{aligned}
$$

Since $\lim _{n=\infty} \sigma\left(x_{n}\right) / \sigma\left(\xi^{\prime}\right)=\lim _{n=\infty} \sigma\left(x_{n}\right) / \sigma\left(x_{n}+\sigma\left(x_{n}\right) \xi\right)=1$ for a.a. $\xi$, we have by Lebesgue's theorem of dominated convergence that the last term is zero. We have established (7) and (8).

It follows from (3) that $x_{n}+\sigma\left(x_{n}\right) x \rightarrow \infty$ as $x_{n} \rightarrow \infty$. We have $\lim _{n=\infty} f_{n}(x)$ $=\lim _{n=\infty} f\left(x_{n}+\sigma\left(x_{n}\right) x\right)=0$, which means that $\lim _{n=\infty}\left(H * f_{n}\right)(x)=0$. By (7), we conclude that

$$
\lim _{n=\infty}\left(K * g_{n}\right)(x)=0 .
$$

Select a subsequence $\left\{g_{n_{v}}\right\}$ of $\left\{g_{n}\right\}$ such that

$$
\begin{gathered}
\lim _{v=\infty} g_{n_{v}}(0)=a \quad \text { with }|a| \geqq d . \\
\lim _{v=\infty} g_{n_{v}}=\psi \quad \text { weakly in } L^{\infty} .
\end{gathered}
$$

The following lemma will guarantee that $\psi$ is uniformly continuous on the $E$ defined in the introduction. Therefore it can be assumed to be uniformly continuous on the real line.

LEMMA. We have

$$
\underset{n=\infty}{\lim \sup }\left|g_{n}(x)-g_{n}(y)\right| \leqq\|\varphi\|_{L^{\infty}} \alpha(x-y) \quad \text { if } x, y \in E .
$$

Proof. Let

$$
x_{n}+\sigma\left(x_{n}\right) x=a_{n}, \quad x_{n}+\sigma\left(x_{n}\right) y=b_{n} .
$$


Then

$$
\begin{aligned}
\mid g_{n}(x)- & g_{n}(y) \mid \\
\leqq & \int_{-\infty}^{\infty}|\varphi(\xi)|\left|H\left(\frac{a_{n}-\xi}{\sigma\left(a_{n}\right)}\right) \frac{1}{\sigma\left(a_{n}\right)}-H\left(\frac{b_{n}-\xi}{\sigma\left(b_{n}\right)}\right) \frac{1}{\sigma\left(b_{n}\right)}\right| d \xi \\
\leqq & \|\varphi(\xi)\|_{L^{\infty}}\left(\int_{-\infty}^{\infty}\left|H\left(\frac{a_{n}-\xi}{\sigma\left(a_{n}\right)}\right) \frac{1}{\sigma\left(a_{n}\right)}-H\left(\frac{b_{n}-\xi}{\sigma\left(a_{n}\right)}\right) \frac{1}{\sigma\left(a_{n}\right)}\right| d \xi\right. \\
& \left.\quad+\int_{-\infty}^{\infty}\left|H\left(\frac{b_{n}-\xi}{\sigma\left(a_{n}\right)}\right) \frac{1}{\sigma\left(a_{n}\right)}-H\left(\frac{b_{n}-\xi}{\sigma\left(b_{n}\right)}\right) \frac{1}{\sigma\left(b_{n}\right)}\right| d \xi\right) \\
= & \|\varphi(\xi)\|_{L^{\infty}}\left(\alpha\left(\frac{a_{n}-b_{n}}{\sigma\left(a_{n}\right)}\right)+\omega\left(\frac{\sigma\left(b_{n}\right)}{\sigma\left(a_{n}\right)}\right)\right) .
\end{aligned}
$$

It follows from our assumption that $\left(a_{n}-b_{n}\right) / \sigma\left(a_{n}\right) \rightarrow x-y$ and $\sigma\left(b_{n}\right) / \sigma\left(a_{n}\right) \rightarrow$ 1 as $n \rightarrow \infty$. Hence our lemma follows.

Now $\psi$ is a nontrivial bounded uniformly continuous function and satisfies $K * \psi=0$. It had been established in [1, p. 134] that the existence of such a $\psi$ implies the Fourier transform of $K$ vanishes at the spectrum of $\psi$, which is not an empty set [1, Theorem 1]. It obviously contradicts our assumptions on $K$. The proof of the theorem is therefore finished.

2. Some applications. It is obvious that Theorem I applies to a wider class of problems than does Wiener's original theorem. A few examples will be demonstrated in the following sequence.

The following theorem about summability had been established in [3].

THEOREM II. Let $\left\{s_{n}\right\}$ be a sequence of bounded numbers. Suppose that

Then it follows

$$
\lim _{x=\infty} \sum_{n=0}^{\infty} s_{n} \frac{x^{n}}{n !} e^{-x}=A
$$

$$
\lim _{n=\infty} \frac{\sum_{n \leqq i<n+\varepsilon \sqrt{ } n} s_{i}}{\varepsilon \sqrt{ } n}=A,
$$

where $\varepsilon$ is any positive real number. Moreover if $s_{n}-s_{n-1}=O(1 / \sqrt{ } n)$ then $\lim _{n=\infty} s_{n}=A$.

Proof. It can be shown easily that if $(x-n)=O(\sqrt{ } x)$ then

$$
\left(x^{n} / n !\right) e^{-x} \sim(1 / \sqrt{ }(2 \pi x)) \exp \left(-(1 / 2)(x-n)^{2} / x\right)
$$

as $x \rightarrow \infty$. Let function $s(u)$ be defined as

$$
\begin{array}{ll}
s(u)=s_{[u]}, & \text { if } u \geqq 0, \\
s(u)=0, & \text { if } u<0 .
\end{array}
$$


Then equation (11) can be replaced by the following integral

$$
\lim _{x=\infty} \int_{-\infty}^{\infty} K\left(\frac{x-n}{\sigma(x)}\right) \frac{1}{\sigma(x)} s(n) d n=A
$$

where $K(x)=(1 / \sqrt{ }(2 \pi)) \exp \left(-{ }_{2}^{1} x^{2}\right), \sigma(x)=\sqrt{ } x$. It is trivial to check that all conditions on $K$ and $\sigma$ are satisfied. It follows that if we let

then we have

$$
\begin{aligned}
H(x) & =1 / \varepsilon, & & \text { if } x \in(-\varepsilon, 0), \\
& =0, & & \text { if } x \notin(-\varepsilon, 0),
\end{aligned}
$$

$$
\lim _{x=\infty} \int_{-\infty}^{\infty} H\left(\frac{x-n}{\sigma(x)}\right) \frac{1}{\sigma(x)} s(n) d n=\lim _{x=\infty} \frac{1}{\varepsilon \sqrt{ } x} \int_{x}^{x+\varepsilon v^{\prime} x} s(n) d n=A
$$

which is the first part of the theorem. The second part follows by the assumption $s_{n}-s_{n-1}=O(1 / \sqrt{ } n)$ on letting $\varepsilon$ tend to 0 in (14).

We finish by an application to bounded solutions $u(x, y)$ in the halfplane $y>0$ of the Laplace equation

$$
\partial^{2} u / \partial x^{2}+\partial^{2} u / \partial y^{2}=0
$$

and of the heat equation

$$
\partial^{2} u / \partial x^{2}-\partial^{2} u / \partial y^{2}=0
$$

Let $\Gamma$ be a Jordan curve in $y>0, \Gamma=\{(x, y) ; y=\sigma(x), 0<x<\infty\}$. If $\varphi(x)$ is the boundary value of $u$ for $y=0$, then $u$ has a representation

with

$$
u(x, y)=\int_{-\infty}^{\infty} \varphi(\xi) K\left(\frac{x-\xi}{\alpha(y)}\right) \frac{1}{\alpha(y)} d \xi
$$

$$
K(x)=(1 / \pi)\left(1 /\left(1+x^{2}\right)\right), \quad \alpha(y)=y,
$$

in the harmonic case, and with

$$
K(x)=(1 / \sqrt{ }(2 \pi)) \exp \left(-x^{2} / 2\right), \quad x(y)=\sqrt{ } y,
$$

in the case of the heat equation. Let us assume that the conditions (3) and (4) are satisfied by the function $\sigma(x)$ (respectively $\sqrt{ }(\sigma(x))$ in the harmonic case (resp. in the case of heat equation). Now we apply Theorems I and II and conclude that if $u(x, y)$ tends to a limit $A$ when $(x, y)$ tends to $\infty$ along $\Gamma$, then for each $\varepsilon>0$ we have

$$
\lim _{x=\infty} \frac{1}{\varepsilon x} \int_{x}^{x+\varepsilon x} \varphi(\xi) d \xi=A
$$


in the harmonic case, and

$$
\lim _{x=\infty} \frac{1}{\varepsilon \sqrt{ } x} \int_{x}^{x+\varepsilon \sqrt{ } x} \varphi(\xi) d \xi=A
$$

in the case of the heat equation.

\section{REFERENCES}

1. A. Beurling, Un théorème sur les fonctions bornées et uniformément continues sur l'axe réel, Acta Math. 77 (1945), 127-136. MR 7, 61.

2. N. Wiener, Tauberian theorem, Ann. of Math. 33 (1932), 1-100.

3. W. B. Jurkat, Ein funktionentheoretischer Beweis für 0-Taubersätze bei den Verfahren non Borel und Euler-Knopp, Arch. Math. 7 (1956), 278-283. MR 18, 479.

School of Mathematics, Institute for Advanced Study, Princeton, New JERSEY 08540

Current address: Department of Mathematics, University of Minnesota, Minneapolis, Minnesota 55455 